\section{Ocorrência de casos de leishmaniose tegumentar americana: uma análise multivariada dos circuitos espaciais de produção, Minas Gerais, Brasil, 2007 a 2011}

\section{American tegumentary leishmaniasis: a multivariate analysis of the spatial circuits for production of cases in Minas Gerais State, Brazil, 2007 to 2011}

\author{
Ocurrencia de casos de leishmaniasis tegumentaria \\ americana: un análisis multivariado de los \\ circuitos espaciales de producción, Minas \\ Gerais, Brasil, 2007 a 2011
}

Andrea Oliveira Dias Temponi 1,2

Mariana Gontijo de Brito 1

Marcela Lencine Ferraz 1

Soraia de Araújo Diniz 2

Marcos Xavier Silva 2

Tarcísio Neves da Cunha ${ }^{3}$

doi: 10.1590/0102-311X00165716

\section{Resumo}

O objetivo deste estudo foi explicar a associação dos fatores socioambientais e dos grandes usos da terra com a ocorrência de casos de leishmaniose tegumentar americana (LTA) nos circuitos espaciais de produção, no Estado de Minas Gerais, Brasil. Trata-se de um estudo ecológico do tipo analítico, baseado em dados secundários de casos de LTA dividido por triênio, no período entre 2007 a 2011, cujas unidades de análise foram os municípios pertencentes aos circuitos espaciais. Duas etapas distintas foram realizadas, sendo a elaboração de mapas temáticos com a identificação dos circuitos a primeira, e na segunda etapa um novo indicador casos de LTA por densidade demográfica foi associado com indicadores socioambientais e dos grandes usos da terra submetidos à análise multivariada de componentes principais (ACP). Para o período avaliado, identificou-se três circuitos distribuídos nas mesorregiões Norte de Minas Gerais, Vale do Rio Doce e Região Metropolitana de Belo Horizonte. Houve forte associação dos casos de LTA por densidade demográfica com lavoura temporária, pastagem natural, floresta natural, terras inaproveitáveis e população rural, e uma fraca associação com pastagem plantada. A associação de casos com variáveis dos grandes usos da terra em diferentes perfis agropecuários demonstra o caráter ocupacional da LTA, associado principalmente com trabalhadores da zona rural. A associação da doença com as variáveis ambientais e deficiência das condições de saneamento básico também demonstram relevância no perfil de transmissão nos circuitos espaciais de produção em Minas Gerais.

Leishmaniose Cutânea; Indicadores Ambientais; Análise Espacial

\author{
Correspondência \\ A. O. D. Temponi \\ Secretaria de Estado de Saúde de Minas Gerais. \\ Rod. Papa João Paulo II 4101, Serra Verde/Prédio Minas/ \\ 13 o andar, Belo Horizonte, MG 31630-900, Brasil. \\ andrea.dias@saude.mg.gov.br \\ 1 Secretaria de Estado de Saúde de Minas Gerais, Belo \\ Horizonte, Brasil. \\ 2 Escola de Veterinária, Universidade Federal de Minas Gerais, \\ Belo Horizonte, Brasil. \\ 3 Escola Nacional de Saúde Pública Sergio Arouca, Fundação \\ Oswaldo Cruz, Rio de Janeiro, Brasil.
}




\section{Introdução}

A leishmaniose tegumentar americana (LTA) é uma doença infecciosa, não contagiosa, causada por protozoários do gênero Leishmania, de transmissão vetorial ${ }^{1}$. De acordo com a Organização Mundial da Saúde (OMS), há aproximadamente de 0,7 a 1,3 milhão de casos por LTA no mundo, distribuídos em 85 países ${ }^{1}$. No Brasil, a LTA apresenta elevada frequência, além de ampla expansão territorial e transmissão essencialmente focal 2. No período entre 2001 e 2010, a doença apresentou um total de 248.834 casos, porém, houve uma tendência à diminuição na última década, em torno de 17,5\% a 20\% em 2010, quando comparado com o ano de 2001 3. No mesmo período, em Minas Gerais registrou-se um total de 15.434 casos novos, distribuídos predominantemente entre os municípios localizados nas regiões Norte de Minas, Vale do Rio Doce, Jequitinhonha e Metropolitana de Belo Horizonte. Dos 853 municípios mineiros, houve registro em 672 (78,7\%), porém, há regiões onde os casos ocorrem de forma mais concentrada, já em outras se apresentam de forma isolada 4.

Nas últimas décadas, análises epidemiológicas têm sugerido mudanças no padrão de transmissão, ocorrendo em zonas rurais praticamente desmatadas, e em regiões periurbanas 1. A dinâmica da transmissão diferencia entre os locais de ocorrência em função das variáveis relacionadas aos parasitos, aos vetores, aos ecossistemas e aos processos sociais de produção e uso do solo 2. Embora as características epidemiológicas e paisagísticas desses espaços sejam semelhantes, elas constituem áreas onde as formas de ocupação do espaço e de uso do solo implicam processos ecológicos e sociais que resultam em instabilidade na incidência da LTA nas localidades que a integram 5 .

A LTA pode ser considerada uma doença ocupacional, com reflexos no campo social e econômico por estar diretamente relacionada com atividades profissionais em áreas enzoóticas 1,6,7. Os casos estão associados principalmente ao sexo masculino em idade produtiva, que exercem atividades de desmatamento e/ou reflorestamento, além de práticas agrícolas, extração de madeira e petróleo, construção de estradas, colheita, caça, pesca, mineração, atividades de pesquisa em florestas tropicais, loteamentos, comunidades adjacentes a florestas, entre outros 7,8. Porém, pode ocorrer em áreas com precárias condições socioeconômicas ${ }^{9}$, com invasão no ambiente doméstico ${ }^{6}$. Destaca-se um número expressivo de casos em mulheres e em crianças em diferentes áreas da América do Sul, sendo este, um indicador de transmissão intra e peridomiciliar 10.

O Ministério da Saúde adota como modelo de vigilância a identificação de áreas prioritárias para ação, prevenção e controle da LTA. Esse modelo utiliza o cálculo de densidade de casos (número de casos de LTA por município de residência, por $\mathrm{km}^{2}$ ) 2. O indicador densidade de casos remete à concentração dos casos em um determinado território e da ideia de localidade da doença 11. Baseando-se nesse cálculo, são elaborados mapas temáticos para períodos de três anos, dos quais se obtém os principais circuitos e polos de produção no Brasil 2.

Ainda, de acordo com o Ministério da Saúde, o conceito de circuito espacial de produção da leishmaniose tegumentar seria uma região extensa, complexa e contínua, definida com base em elevada concentração de casos em um período de três anos, sendo constituído por diversos polos, superpondo a mais de um município ou estado. Os circuitos podem ser decorrentes de processos socioambientais, particulares e dinâmicos, e apresentar tendência à expansão ou retração, em função das características de seus determinantes. Já um polo é uma unidade espacial que apresenta intensa densidade de casos em contraste com áreas vizinhas, de limites pouco definidos, dinâmico, e do ponto de vista epidemiológico possivelmente apresenta características diferenciadas do restante da sua região 2.

Considerando-se o cenário epidemiológico da LTA no estado, faz-se necessário conhecer a implicação da manutenção nas áreas com maior ocorrência da doença. Há poucos trabalhos que apresentem uma abordagem espacial associada aos fatores de risco no território mineiro, como o estudo realizado na região sudeste de Minas Gerais, entre 1966 e 1996, cujo objetivo foi avaliar a ocorrência espaço temporal da LTA e fatores de risco associados à sua transmissão 12 . As informações a respeito da distribuição espacial são importantes, pois permitem formular hipóteses sobre os principais determinantes, além de possibilitar a organização e o planejamento das ações de saúde e controle do vetor.

Este trabalho tem como objetivo identificar os circuitos espaciais de produção, utilizando como base o modelo de vigilância e monitoramento da LTA no Brasil, aplicado para a realidade de Minas Gerais, e avaliar os fatores socioambientais e dos grandes usos da terra associados com a ocorrência de casos de LTA em áreas de maior densidade de casos entre 2007 a 2011. 


\section{Material e métodos}

\section{Área de estudo}

O Estado de Minas Gerais está localizado na Região Sudeste do Brasil; se situa geograficamente entre os paralelos $14^{\circ} 13^{\prime} 58^{\prime \prime}$ e $22^{\circ} 55^{\prime} 22^{\prime \prime}$ de latitude sul e os meridianos $30^{\circ} 51^{\prime} 23^{\prime \prime}$ e $51^{\circ} 02^{\prime} 45^{\prime \prime}$ de longitude oeste. A área territorial total oficial é de $586.222,122 \mathrm{~km}^{2}$. Faz limites ao norte e nordeste com a Bahia, a leste com o Espírito Santo, a sudeste com o Rio de Janeiro, a sudoeste e sudeste com São Paulo, a oeste com Mato Grosso do Sul e, a noroeste, com Goiás e Distrito Federal. Possui 853 municípios, sua população é de 19.597.330 habitantes e a densidade demográfica é de 33,41 habitantes por km² 13 . Aproximadamente $85,3 \%$ da população vivem nos centros urbanos e $49,2 \%$ são do sexo masculino (Departamento de Informática do SUS. http://tabnet.datasus.gov.br/cgi/deftohtm.exe?ibge/cnv/ popMG.def, acessado em 30/Mar/2014).

O estudo restringiu-se principalmente aos municípios da Mesorregião Metropolitana de Belo Horizonte, do Vale do Rio Doce e do Norte de Minas, em função da disposição dos circuitos espaciais de LTA no estado.

\section{- Municípios pertencentes aos circuitos espaciais de produção}

Foi obtida da página de Internet do projeto Monitoramento e Vigilância da Leishmaniose Tegumentar no Brasil (http://www4.ensp.fiocruz.br/Leishmaniose/lt/) a seleção dos municípios pertencentes aos circuitos de Minas Gerais, para os triênios 2007 a 2009 (38 municípios), 2008 a 2010 (61 municípios) e, por fim, 2009 a 2011 (49 municípios), sendo este triênio o último levantamento realizado pelo Ministério da Saúde.

\section{Delineamento do estudo}

Trata-se de um estudo epidemiológico ecológico do tipo analítico, no qual objetiva-se avaliar os aspectos socioambientais e do uso da terra na transmissão da LTA, por meio da caracterização de circuitos espaciais de transmissão, no período entre 2007 e 2011 em Minas Gerais.

\section{Procedimentos metodológicos}

Os procedimentos metodológicos deste trabalho envolveram duas distintas etapas. A primeira foi a elaboração de mapas temáticos para a identificação dos circuitos espaciais e; na segunda etapa, um novo indicador casos LTA por densidade demográfica foi associado com indicadores socioambientais e dos grandes usos da terra, e submetidos à análise multivariada de componentes principais (ACP), para avaliar como estes fatores podem influenciar a ocorrência de casos nos circuitos.

\section{Fonte da dados}

\section{- Casos de leishmaniose tegumentar}

Para a realização do presente estudo foram utilizados dados secundários de casos novos de LTA, segundo o município de residência, do Sistema de Informação de Agravos de Notificação (SINAN), versão SINAN Net 4.0, obtidos junto à Secretaria de Estado de Saúde de Minas Gerais (SES-MG).

O período do estudo compreendeu os anos de 2007 (1.349 casos), 2008 (1.183), 2009 (1.050 casos), 2010 (1.939) e 2011 (1.782 casos), agrupados em intervalos de três anos, totalizando 7.303 casos em todo o estado. O primeiro intervalo foi composto pelos anos de 2007 a 2009 (3.582 casos acumulados), o segundo compreendeu os anos de 2008 a 2010 (4.182) e o terceiro de 2009 a 2011 (4.771 casos). Os municípios pertencentes aos circuitos apresentaram no total 1.187 casos entre 2007 e 2009, 1.492 de 2008 a 2010 e 1.593 casos entre 2009 e 2011. 


\section{- Geração de mapas}

Para a elaboração dos mapas de densidade foi utilizado o programa MapInfo Pro versão 12.0 (MapInfo Corp., Nova York, Estados Unidos). O primeiro passo foi a montagem de uma tabela georreferenciada contendo todos os municípios de Minas Gerais. Cada município recebeu os valores de densidade média de LTA para os triênios: (a) 2007 a 2009; (b) 2008 a 20010; e (c) 2009 a 2011.

Os municípios apresentam grande diversidade de extensão territorial e forma geométrica, o que dificulta a regionalização da densidade de casos por $\mathrm{km}^{2}$. A solução para o geoprocessamento foi interpolar os valores para uma grade regular. No MapInfo isso é denominado de mapa temático do tipo "Grid".

O estado tem um retângulo envolvente de aproximadamente $1.170 \mathrm{~km}$ no sentido leste-oeste e $960 \mathrm{~km}$ no sentido norte-sul. Para uma cobertura composta por 100 células regulares no sentido leste-oeste a largura da célula teria aproximadamente $12 \mathrm{~km}$, o que permitiu cobrir com 104 x 81 células regulares de $12 \mathrm{~km}$ de lado.

O interpolador utilizado para determinar o valor regionalizado das densidades em cada célula foi o inverso do quadrado da distância (IDW no MapInfo) com um raio de busca de $1.200 \mathrm{~km}$, suficiente para que os municípios sejam considerados para o cálculo de cada célula. Nesse procedimento, cada célula recebe a soma das contribuições de todos os municípios. Cada município contribui com o seu valor de densidade dividido pelo quadrado da sua distancia à célula da vez. Dessa forma, a contribuição de cada município cai exponencialmente com a sua distância à célula considerada 14 .

Para delimitar os circuitos, os mapas de grade foram coloridos de acordo com faixas de densidade pré-estabelecidas, o que permitiu que os circuitos fossem desenhados diretamente sobre os mapas digitais com base na cor. A delimitação dos circuitos foi realizada de acordo com o valor de corte adotado para densidades maiores do que 10 casos por $\mathrm{km}^{2}$, segundo o Programa Nacional das Leishmanioses.

\section{- Variáveis socioambientais e do uso da terra}

Para a avaliação dos circuitos presentes na segunda etapa do estudo foi criada uma nova variável, que calcula os casos novos de LTA por densidade demográfica. Os dados foram obtidos do Censo Demográfico de 2010 do Instituto Brasileiro de Geografia e Estatística 13.

Foram utilizados dados do Censo Demográfico de 2010 para estimar a população humana (rural e urbana). Obteve-se informações sobre o uso da terra por meio do Censo Agropecuário de 2006 (lavouras permanentes, lavouras temporárias, pastagens naturais, pastagens plantadas, floresta plantada, matas e florestas naturais, terras inaproveitáveis) 15 .

As variáveis de natureza socioambientais e de condições de infraestrutura urbana, de renda e grau de instrução da população afetada foram obtidas no Índice Mineiro de Responsabilidade Social (IMRS), cujo ano base foi 2010, por meio dos indicadores: percentual da população com banheiro e coleta de esgoto, percentual da população atendida por sistema de coleta e tratamento de lixo, renda per capita e percentual de analfabetismo da população de 10 anos e mais (Fundação João Pinheiro. http://fjp.mg.gov.br/index.php/produtos-e-servicos1/2741-indice-mineiro-de-responsa bilidade-social-imrs-2, acessado em 17/Abr/2014). Também se obtiveram dados do Índice FIRJAN de Desenvolvimento Municipal (IFDM), ano base de 2010 (Sistema FIRJAN. http://www.novomilenio. inf.br/baixada/bsfotos/IFDM2010.pdfa, acessado em 10/Abr/2014).

\section{Análise dos dados}

O método estatístico utilizado para análise da ocorrência de casos novos de LTA e fatores socioambientais e do uso da terra foi a análise multivariada por meio da ACP, com o auxílio do programa estatístico Stata versão 12.0 (StataCorp LP, College Station, Estados Unidos). É uma análise não inferencial, multifatorial de caráter essencialmente descritivo ${ }^{16}$. A construção de componentes principais ocorre por representação gráfica do sistema de coordenadas geográficas. As combinações não são correlacionadas entre si e a suposição de normalidade não é requisito necessário para a sua realização 17,18 . 
Uma boa representação do fenômeno é alcançada se a inércia total do sistema atingir pelo menos $70 \%$, o que por consequência significa que o sistema tridimensional estudado explica $70 \%$ da variação existente nos dados. Quanto mais alto o valor absoluto do coeficiente associado a uma variável, maior a associação relativa desta para o componente principal em questão, podendo esta importância dar-se em termos positivos ou negativos. Duas variáveis estarão muito associadas se elas apresentarem no mesmo quadrante e reduzida distância entre si. Variáveis em quadrantes opostos irão exprimir associação negativa, e fraca associação se situadas em quadrantes vizinhos. As variáveis mais importantes serão as que apresentarem maiores distâncias em relação à origem 16 .

\section{Aspectos éticos}

O estudo foi aprovado pelo Comitê de Ética em Pesquisa da Universidade Federal de Minas Gerais (UFMG; CAAE protocolo 30992614.6.0000.5149).

\section{Resultados}

A avaliação dos três circuitos espaciais nos triênios 2007-2009, 2008-2010 e 2009-2011 detiveram aproximadamente $33,1 \%, 35,8 \%$ e $33,4 \%$ do total de casos notificados no estado.

A distribuição espacial dos circuitos por triênios é observada por meio de três grandes áreas com maior relevância epidemiológica, que apresentaram maior concentração de casos por $\mathrm{km}^{2}$. As áreas mais quentes identificadas no mapa estão distribuídas entre as mesorregiões Metropolitana de Belo Horizonte, Vale do Rio Doce e Norte de Minas (Figura 1).

A partir do triênio 2008-2010, observa-se uma dispersão da doença no estado, com destaque para as áreas em amarelo que apresentaram uma densidade média em torno de 5 casos por km². Destacase também a expansão das áreas de transmissão presentes nos Circuitos 1 e 2, que apresentaram um maior número de municípios com densidade média acima de 10 casos por $\mathrm{km}^{2}$.

O mesmo pode ser observado para o triênio 2009-2011, que apresentou ampliação da doença para os municípios presentes nas mesorregiões Norte de Minas, Jequitinhonha, Vale do Mucuri e Vale do Rio Doce, com registro geral de até 8 casos por $\mathrm{km}^{2}$, identificados pelas áreas em amarelo e verde no mapa. Essa dispersão mostra que a doença assumiu dimensões epidêmicas nesse período. Esse comportamento da doença pode ser observado nos municípios de Patos de Minas em 2010, que registrou 77 casos novos (média 2007-2009: 4 casos) e Paracatu com 50 casos (média 2007-2009: 6 casos), localizados na mesorregião do Triângulo Mineiro/Alto Paranaíba e Noroeste de Minas, respectivamente; e no município de Minas Novas em 2011, localizado na mesorregião do Jequitinhonha, que apresentou um registro de 48 casos (média 2007-2009: 2 casos).

Na Tabela 1, observam-se as coordenadas das variáveis que definem a localização das mesmas no sistema algébrico tridimensional. Também observa-se o resultado de inércia explicada e acumulada para os circuitos espaciais, referente aos casos de LTA segundo densidade demográfica com as variáveis socioambientais e variáveis do uso da terra.

O sistema apresentou um resultado satisfatório, pois apresentou valor de inércia acumulada para os três primeiros eixos de 73\% na avaliação geral (Circuitos 1, 2 e 3), de $81 \%$ para o Circuito 1 e de $78 \%$ para o Circuito 2. Não houve robustez de observações para a avaliação do Circuito 3 por meio da ACP.

Os dados gerados com base no banco de dados possibilitaram determinar os seguintes gráficos de dispersão para estudo, representados nas Figuras 2, 3 e 4, que dispõem a localização gráfica das variáveis no plano cartesiano tridimensional.

Na Figura 2, observa-se avaliação geral por meio da ACP para os três circuitos (1, 2 e 3). A variável casos por densidade demográfica por estar disposta no mesmo quadrante e com reduzida distância entre lavoura temporária, terras inaproveitáveis, floresta natural, pastagem natural e população rural apresentou alta associação e uma relativa associação com pastagem plantada. As variáveis que apresentaram fraca associação entre si por se apresentarem em quadrantes vizinhos foram o IFDM, assim como os subíndices do IMRS representados pela taxa de analfabetismo em maiores de 10 anos, percentual da população com banheiro e coleta de esgoto, percentual da população atendida por sistema de coleta e tratamento de lixo e renda per capita. As variáveis população urbana e lavoura permanente 
Figura 1

Densidade média de casos de leishmaniose tegumentar americana por área (km²) e circuitos espaciais de produção. Minas Gerais, Brasil, 2007 a 2011.

1a) 2007-2009
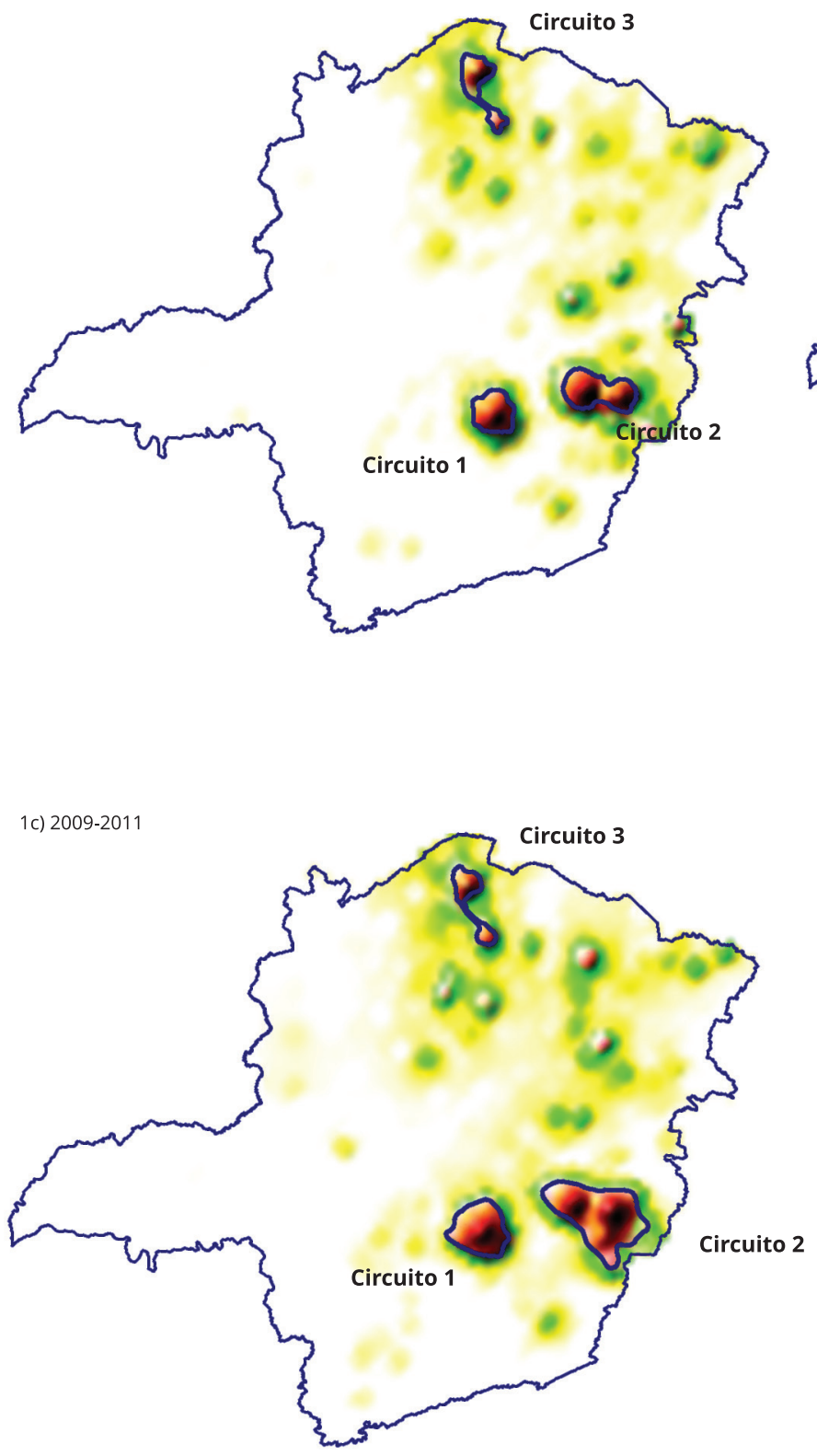

1b) $2008-2010$

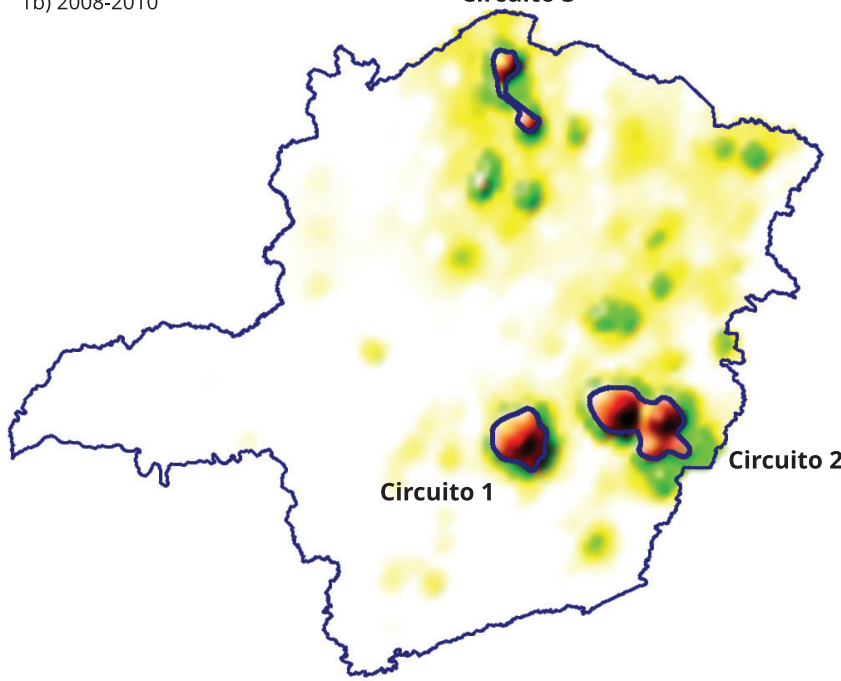

Densidade média de casos por $\mathrm{km}^{2}$ (x 1.000)

5

8

$\square 12$

$\square 20$

$\square 50$

$\square 100$

500

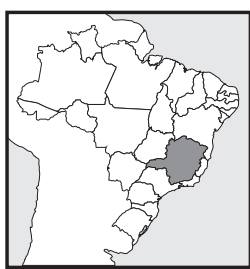

também apresentaram fraca associação com a variável casos de LTA por densidade demográfica. Não houve disposição oposta entre as variáveis estudadas com casos por densidade demográfica para o período e os três circuitos avaliados.

$\mathrm{Na}$ avaliação do Circuito 1 (Figura 3), destaca-se o aumento do percentual de inércia acumulada para $81 \%$. Houve associação da variável de casos por densidade demográfica com as variáveis floresta plantada, população rural, lavoura permanente e pastagem natural. 


\section{Tabela 1}

Coordenadas das variáveis estudadas, valor da inércia explicada e acumulada da análise multivariada de componentes principais dos casos de leishmaniose tegumentar americana, por densidade demográfica com as variáveis socioambientais e do uso da terra, segundo circuitos espaciais de produção. Minas Gerais, Brasil, 2007 a 2011.

\begin{tabular}{|c|c|c|c|c|c|c|c|c|c|}
\hline \multirow[t]{2}{*}{ Variáveis } & \multicolumn{3}{|c|}{ Componente principal 1} & \multicolumn{3}{|c|}{ Componente principal 2} & \multicolumn{3}{|c|}{ Componente principal 3} \\
\hline & $\begin{array}{c}\text { Circuitos } \\
1,2 \text { e } 3\end{array}$ & $\begin{array}{c}\text { Circuito } \\
1\end{array}$ & $\begin{array}{c}\text { Circuito } \\
2\end{array}$ & $\begin{array}{c}\text { Circuitos } \\
1,2 \text { e } 3\end{array}$ & $\begin{array}{c}\text { Circuito } \\
1\end{array}$ & $\begin{array}{c}\text { Circuito } \\
2\end{array}$ & $\begin{array}{c}\text { Circuitos } \\
1,2 \text { e } 3\end{array}$ & $\begin{array}{c}\text { Circuito } \\
1\end{array}$ & $\begin{array}{c}\text { Circuito } \\
2\end{array}$ \\
\hline $\begin{array}{l}\text { Casos/Densidade } \\
\text { demográfica }\end{array}$ & 0,328 & 0,215 & 0,328 & 0,162 & 0,295 & $-0,125$ & $-0,277$ & $-0,245$ & 0,010 \\
\hline Lavoura permanente & 0,086 & 0,320 & 0,263 & $-0,035$ & 0,105 & 0,135 & 0,741 & 0,036 & $-0,312$ \\
\hline Lavoura temporária & 0,363 & 0,320 & 0,358 & 0,146 & $-0,087$ & $-0,095$ & $-0,067$ & 0,218 & $-0,046$ \\
\hline Pastagem natural & 0,274 & 0,311 & 0,363 & 0,112 & 0,094 & $-0,060$ & 0,418 & 0,297 & $-0,105$ \\
\hline Pastagem plantada & 0,354 & 0,296 & 0,321 & 0,161 & $-0,044$ & $-0,135$ & $-0,012$ & 0,385 & $-0,039$ \\
\hline Floresta natural & 0,350 & 0,303 & 0,338 & 0,205 & $-0,013$ & $-0,054$ & $-0,126$ & 0,386 & 0,352 \\
\hline Floresta plantada & 0,056 & 0,176 & 0,092 & $-0,054$ & 0,436 & $-0,091$ & 0,053 & $-0,175$ & 0,708 \\
\hline Terras inaproveitáveis & 0,333 & 0,311 & 0,343 & 0,210 & $-0,143$ & 0,084 & $-0,189$ & 0,094 & $-0,186$ \\
\hline População urbana & $-0,132$ & $-0,153$ & 0,120 & 0,369 & $-0,304$ & 0,440 & $-0,156$ & 0,317 & 0,102 \\
\hline População rural & 0,331 & 0,070 & 0,358 & 0,152 & 0,443 & 0,053 & 0,257 & 0,157 & $-0,214$ \\
\hline IFDM 10 & $-0,214$ & $-0,282$ & 0,106 & 0,430 & 0,150 & 0,389 & 0,070 & 0,398 & 0,220 \\
\hline IMRS banheiro/esgoto & $-0,233$ & $-0,255$ & $-0,091$ & 0,283 & $-0,059$ & 0,337 & $-0,031$ & 0,140 & 0,099 \\
\hline IMRS lixo & $-0,177$ & $-0,264$ & $-0,168$ & 0,163 & 0,334 & 0,288 & 0,154 & 0,193 & $-0,310$ \\
\hline IMRS renda per capita & $-0,138$ & $-0,126$ & 0,173 & 0,436 & 0,495 & 0,414 & 0,146 & 0,137 & 0,134 \\
\hline IMRS analfabetismo & 0,198 & 0,295 & 0,006 & $-0,428$ & $-0,046$ & $-0,449$ & $-0,028$ & $-0,327$ & $-0,017$ \\
\hline Inércia explicada (\%) & 0,43 & 0,56 & 0,42 & 0,210 & 0,15 & 0,26 & 0,090 & 0,09 & 0,09 \\
\hline Inércia acumulada (\%) & 0,43 & 0,56 & 0,42 & 0,64 & 0,71 & 0,68 & 0,73 & 0,81 & 0,78 \\
\hline
\end{tabular}

IFDM: Índice FIRJAN de Desenvolvimento Municipal; IMRS: Índice Mineiro de Responsabilidade Social.

Foi identificada também uma fraca associação com a variável pastagem plantada, além dos subíndices do IMRS (percentual da população atendida por sistema de coleta e tratamento de lixo, e taxa de analfabetismo em maiores de 10 anos), com o IFDM, lavoura temporária, terras inaproveitáveis e floresta natural. Nesse sistema, foi identificada associação negativa entre a ocorrência de casos de LTA por densidade demográfica com população urbana e condições de saneamento, representadas pelo subíndice do IMRS percentual da população com banheiro e coleta de esgoto.

Na avaliação do Circuito 2 (Figura 4), houve um aumento do percentual de inércia acumulada de 73\% para 78\% quando comparado com a análise geral (Circuitos 1, 2 e 3).

Houve uma forte associação da variável de casos de LTA por densidade demográfica com as variáveis de pastagem plantada, pastagem natural, lavoura temporária, floresta natural e floresta plantada. A variável floresta plantada, apesar de estar no mesmo quadrante e apresentar associação com a variável casos, ocorreu de forma menos expressiva do que as demais variáveis. Esse resultado também foi semelhante com a variável do subíndice do IMRS taxa de analfabetismo em maiores e 10 anos, que se destacou por apresentar uma curta distância em relação à origem e uma grande distância entra a variável casos.

A associação positiva encontrada no Circuito 2 demonstra a relação do risco da transmissão da doença relacionado à floresta e ao ambiente agrícola. Uma fraca associação foi observada para as variáveis do IFDM, população urbana, população rural, pelo subíndice do IMRS renda per capita, terras inaproveitáveis e, por fim, a variável lavoura permanente.

Uma associação inversa foi identificada entre a variável casos de LTA por densidade demográfica com as variáveis do subíndice IMRS (percentual da população com banheiro e coleta de esgoto, e percentual da população atendida por sistema de coleta e tratamento de lixo). 
Figura 2

Relação dos casos de leishmaniose tegumentar americana com as variáveis socioambientais e das variáveis do uso da terra nos circuitos espaciais de produção. Minas Gerais, Brasil, 2007 a 2011.

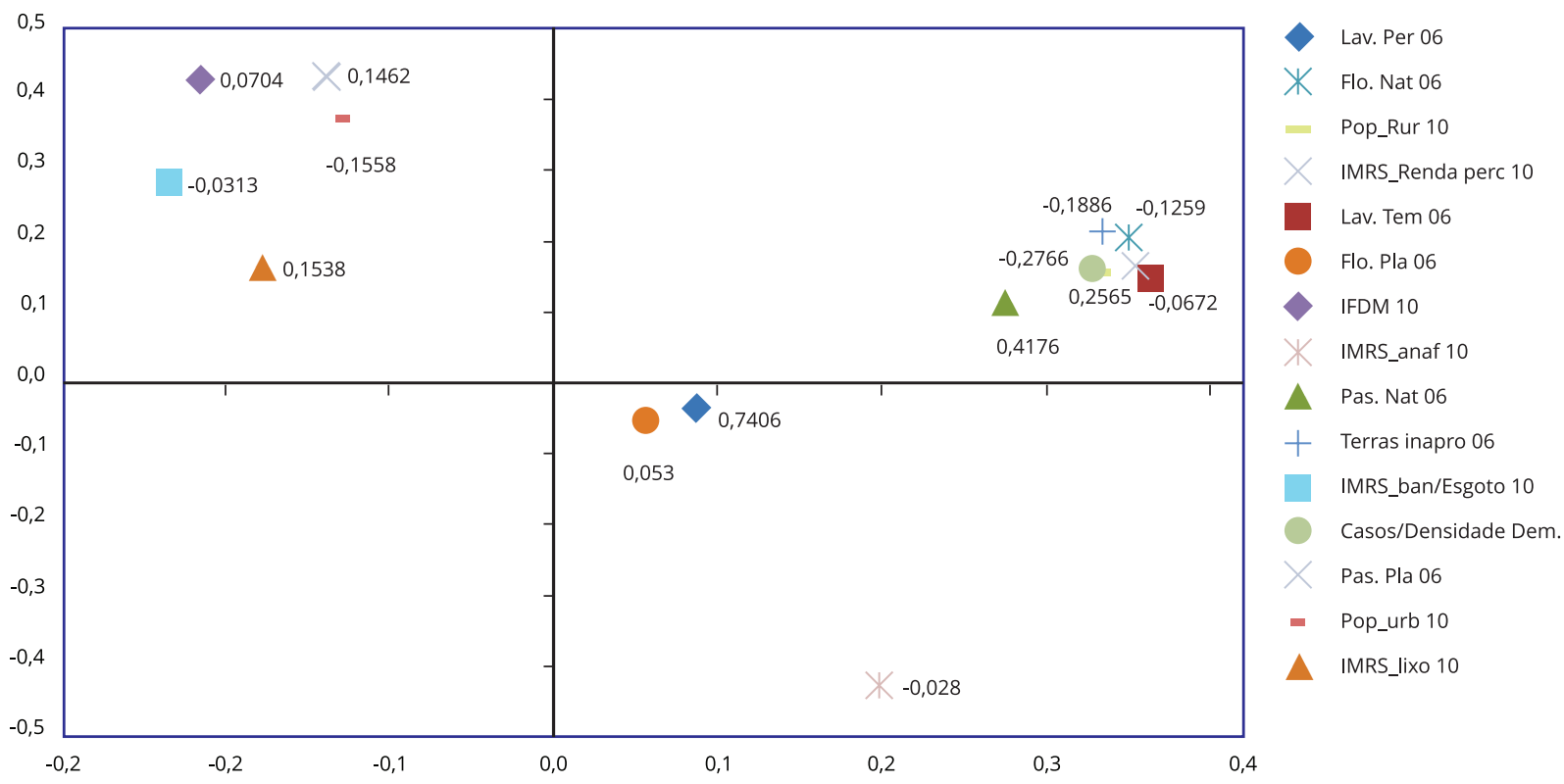

Casos/Densidade Dem.: casos/densidade demográfica; Flo. Nat: floresta natural; Flo. Pla: floresta plantada; IFDM: Índice FIRJAN de Desenvolvimento Municipal; IMRS: Índice Mineiro de Responsabilidade Social; IMRS_anaf: IMRS analfabetismo; IMRS_ban/Esgoto: IMRS banheiro/esgoto; IMRS_Renda perc: IMRS renda per capita; Lav. Per: lavoura permanente; Lav. Tem: lavoura temporária; Pas. Nat: pastagem natural; Pas. Pla: pastagem plantada; Pop_Rur: população rural; Pop_urb: população urbana; Terras inapro: terras inaproveitáveis.

Nota: as coordenadas do eixo 3 estão apostas ao lado da identificação.

\section{Discussão}

Os nossos resultados demonstram que nas áreas com maior transmissão da doença, identificadas pelos circuitos espaciais de produção, reforçam o caráter focal da LTA e as diferenças regionais quanto à transmissão no estado no decorrer do tempo. Esse perfil foi semelhante ao estudo realizado na Venezuela, onde identificou cinco clusters espaço-temporais de LTA estatisticamente significativos $(\mathrm{p}<0,01)^{8}$.

É importante destacar a ocorrência de uma distribuição heterogênea dos casos, com focos descontínuos e de surtos epidêmicos, como ocorreu nos municípios de Patos de Minas e Paracatu em 2010, e Minas Novas em 2011, localizados em áreas que fogem dos limites dos circuitos. Esse comportamento corrobora o trabalho realizado no Rio de Janeiro no que se refere à formação de microfocos circunscritos e transitórios 5 .

Este estudo buscou trabalhar a combinação de diferentes bases de dados por meio da análise multivariada de ACP, no intuito de explicar a associação entre a ocorrência dos casos de LTA com os fatores socioambientais e do uso da terra, considerando-se a hipótese de a permanência da doença nos circuitos sofrer influência de diversos fatores de natureza diversificada. O mesmo modelo foi aplicado em Minas Gerais para a avaliação de casos de síndrome cardiopulmonar por hantavirose e os grandes usos da terra, com valor de inércia acumulada de 79\% 19; e em Belo Horizonte, para a avaliação da incidência de tuberculose com os índices de desenvolvimento humano e indicadores de vulnerabilidade familiar para os anos de 1991 e 2000. O modelo foi satisfatório tanto para o ano de 1991 (84\%) quanto para o ano 2000 (79\%) 16. 
Figura 3

Relação dos casos de leishmaniose tegumentar americana com as variáveis

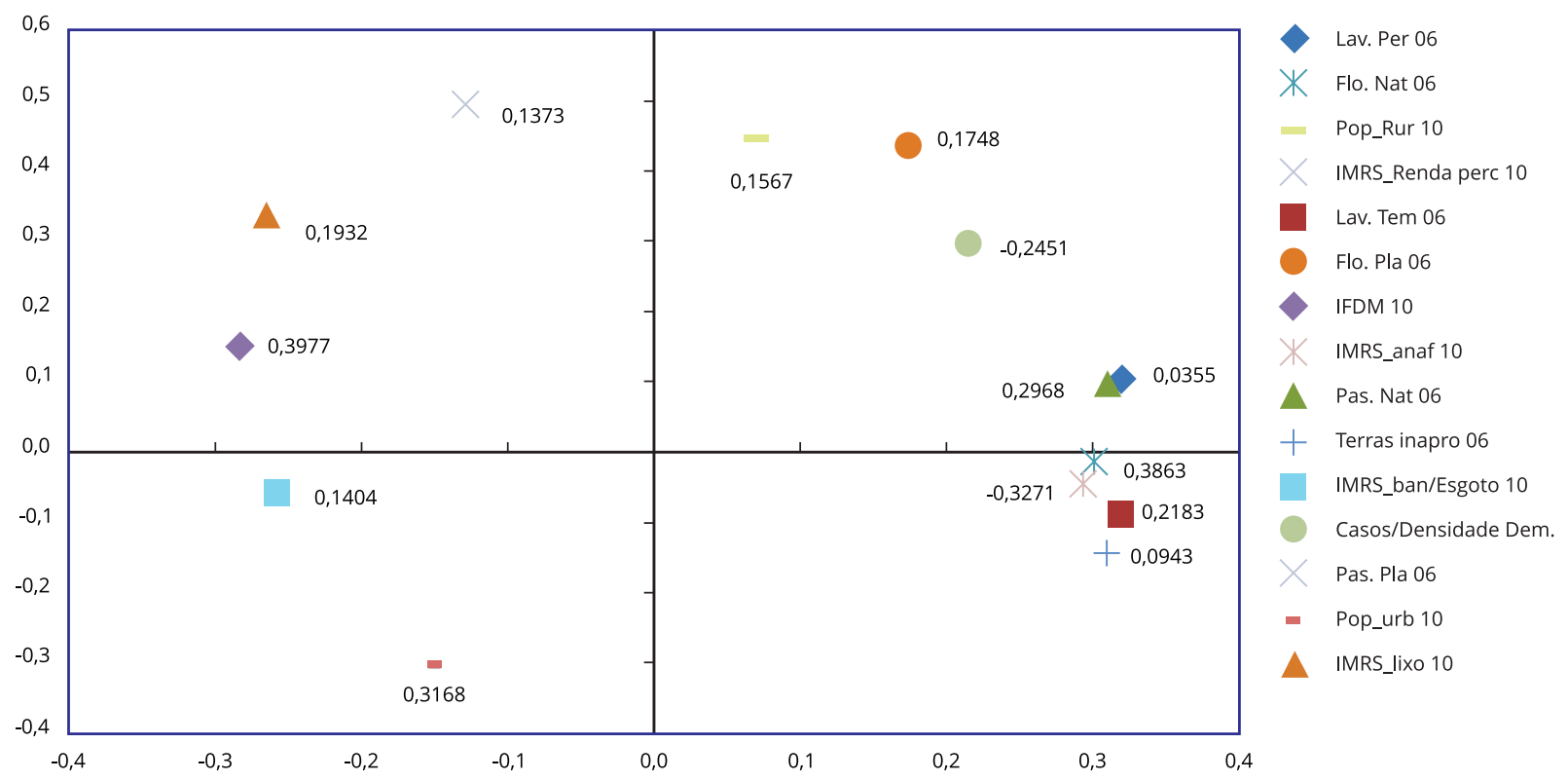

CCasos/Densidade Dem.: casos/densidade demográfica; Flo. Nat: floresta natural; Flo. Pla: floresta plantada; IFDM: Índice FIRJAN de Desenvolvimento Municipal; IMRS: Índice Mineiro de Responsabilidade Social; IMRS_anaf: IMRS analfabetismo; IMRS_ban/Esgoto: IMRS banheiro/esgoto; IMRS_Renda perc: IMRS renda per capita; Lav. Per: lavoura permanente; Lav. Tem: lavoura temporária; Pas. Nat: pastagem natural; Pas. Pla: pastagem plantada; Pop_Rur: população rural; Pop_urb: população urbana; Terras inapro: terras inaproveitáveis.

A análise geral dos circuitos (Circuitos 1, 2 e 3) pela ACP demonstrou uma associação da doença com atividades rurais, áreas de floresta e também com terras inaproveitáveis. Esse resultado mostra o caráter ocupacional da doença nesses territórios, a relação em que o ambiente silvestre alterado pelas práticas agrícolas em áreas rurais pode determinar a ocorrência de casos. Nossos resultados são consistentes com os encontrados em trabalhos anteriores, que também identificaram o ambiente rural 8,12 e áreas de floresta favoráveis para focos de LTA 7,8,20,21.

A proximidade ou contato em áreas com cobertura vegetal, representadas pelos indicadores floresta natural e floresta plantada, também registraram estreita relação com a transmissão, representada na avaliação geral dos Circuitos 1, 2 e 3 com floresta natural, na avaliação do Circuito 1 com floresta plantada e, por fim, na avaliação do Circuito 2 com floresta natural e floresta plantada. As nossas obeservações corroboram o estudo realizado em Tolima, na Colômbia, onde a presença de floresta ou arbusto apresentou um risco de transmissão de LTA de 2,6\% (IC95\%: 0,5-4,9\%) para cada percentual a mais de cobertura vegetal 21.

A associação com terras inaproveitáveis pode ser justificada, segundo o conceito utilizado pelo Censo Agropecuário, que considera como áreas imprestáveis para a formação de culturas, pastos e matas, tais como areais, pântanos, encostas íngremes, pedreiras, estradas, caminhos, construções, canais de irrigação e açudes 15. Considerando-se o conceito, a presença de encostas íngremes e pedreiras podem se destacar por serem propícias para a manutenção da fauna flebotomínica, com relatos da ocorrência de casos em domicílios situados próximos a paredões rochosos 22,23.

A importância do uso da terra por meio da análise dos indicadores do Censo Agropecuário usados neste trabalho (lavoura permanente ou lavoura temporária) e na utilização de pastagens (natural ou plantada) mostrou-se associada com a variável casos por densidade demográfica, seja na avaliação geral dos Circuitos (1, 2 e 3) e também na avaliação dos Circuitos 1 e 2, respectivamente. A identifica- 
Figura 4

Relação dos casos de leishmaniose tegumentar americana com as variáveis socioambientais e das variáveis do uso da terra no Circuito Espacial de Produção 2. Minas Gerais, Brasil, 2007 a 2011.

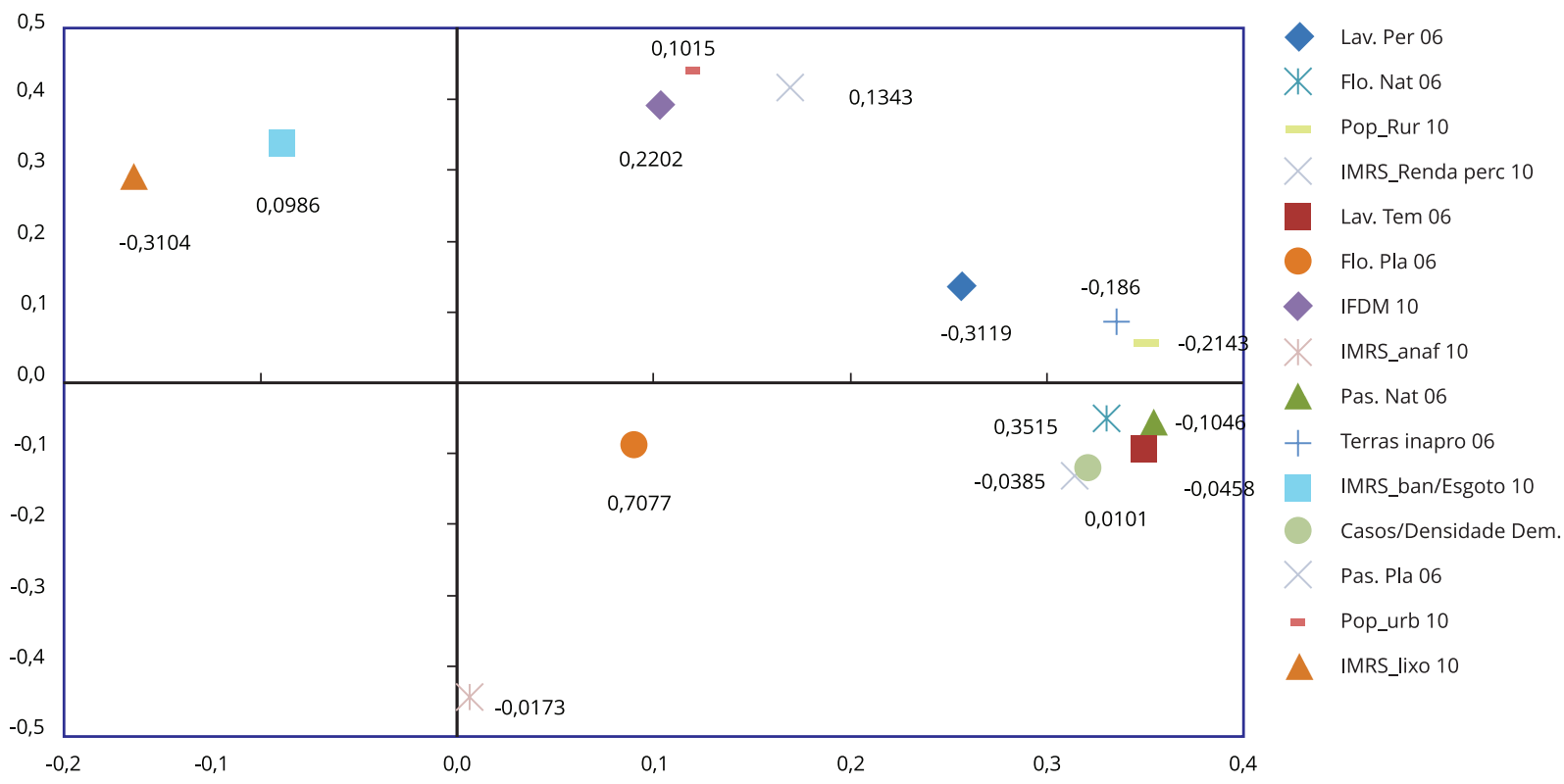

Casos/Densidade Dem.: casos/densidade demográfica; Flo. Nat: floresta natural; Flo. Pla: floresta plantada; IFDM: Índice FIRJAN de Desenvolvimento Municipal; IMRS: Índice Mineiro de Responsabilidade Social; IMRS_anaf: IMRS analfabetismo; IMRS_ban/Esgoto: IMRS banheiro/esgoto; IMRS_Renda perc: IMRS renda per capita; Lav. Per: lavoura permanente; Lav. Tem: lavoura temporária; Pas. Nat: pastagem natural; Pas. Pla: pastagem plantada; Pop_Rur: população rural; Pop_urb: população urbana; Terras inapro: terras inaproveitáveis.

Nota: as coordenadas do eixo 3 estão apostas ao lado da identificação.

ção de diferentes sistemas agropecuários reforça mais uma vez o caráter ocupacional, e como as ações do homem sobre a terra podem afetar a qualidade da paisagem e consequentemente afetar a composição da fauna flebotomínica, podendo ser determinantes na transmissão da LTA nessas regiões 7,20,21.

Não houve associação da variável casos por densidade demográfica com IFDM e com os subíndices do IMRS representados pela taxa de analfabetismo em maiores de 10 anos e renda per capita. Essa associação poderia remeter que uma baixa escolaridade e baixa renda da população reforçariam que a LTA, assim como a maioria das doenças infecto-parasitárias, predomina principalmente em populações mais carentes, o que foi descrito por outros autores 24,25, como no estudo realizado na Costa Rica, onde uma correlação positiva entre a marginalidade e a taxa da doença $(p<0,0002)$ foi identificada 7 . Entretanto, é possível inferir que o desempenho insatisfatório no estudo pode ser devido a variações quantitativas positivas dos indicadores socioeconômicos de renda per capita e taxa de analfabetismo em Minas Gerais, que seguem a mesma tendência nacional 13,26.

No que se refere aos indicadores de qualidade de vida, a relação inversa identificada com a variável casos por densidade demográfica, por meio do esgotamento sanitário (Circuitos 1 e 2) e coleta de lixo (Circuito 2), sugere que a indisponibilidade destes serviços pode proporcionar condições favoráveis à proliferação de vetores e presença de reservatórios no peridomicílio e, consequentemente, aumentar as taxas de LTA. Resultados semelhantes corroboram o nosso resultado como, por exemplo, no estudo realizado em Caratinga (Minas Gerais) 12, onde a transmissão da LTA estava associada a precárias instalações sanitárias e exposição ao lixo ( $<$ <,001). No Município de Teresina (Piauí), a mesma relação foi identificada, porém, com casos de leishmaniose visceral 24 , cuja incidência urbana estava relacionada ao percentual médio de casas sem água tratada $(\mathrm{p}<00,1)$ e, por fim, no norte da Argentina o aumento da prevalência da doença estava relacionado a bairros com esgotamento sanitário deficiente $(\mathrm{p}<0,05) 10$. 
Destaca-se o Circuito 1 que não apresentou associação inversa entre a ocorrência de casos por densidade demográfica e coleta de lixo, o que pode ser justificado porque a maioria dos municípios que o compõe conta com o serviço para grande parte de sua população. Houve associação inversa de casos por densidade demográfica com a população urbana, sendo possível inferir que a introdução de um grande contingente populacional por meio de uma ocupação desordenada, seja de encostas ou de periferias num curto espaço de tempo, pode favorecer a formação de indivíduos expostos a condições ambientais favoráveis ao desenvolvimento do ciclo da LTA. Além disso, um possível processo de domesticação do vetor e a participação de animais domésticos e sinantrópicos como roedores podem servir como reservatórios da doença 27,28,29. Esse cenário também retrata a mudança do perfil de transmissão, que após a década de 1940 com a progressão da derrubada de matas, haveria diminuição da criação de insetos transmissores, o que resultaria na queda do número de casos da doença, entretanto, observou-se após a década de 1980 casos autóctones em novas áreas e em forma de endemia na periferia de centros urbanos, como tem sido constatado na Região Metropolitana de Belo Horizonte 9 .

O Circuito 2, ao contrário, não demonstrou associação da variável casos com a população urbana e a população rural, o que pode ser justificado considerando-se a realidade dos municípios pertencentes à mesorregião do Vale do Rio Doce apresentarem população em torno de 15 mil habitantes. Por serem municípios de pequeno porte, pode haver uma difícil definição das fronteiras entre as zonas urbana e rural, além da sobreposição entre a vegetação urbana e mata residual.

Apesar de não ter havido robustez dos dados para avaliação do Circuito 3 devido à baixa repetitividade dos municípios para avaliação da ACP 17, pode-se inferir que a ocorrência de casos poderia sofrer influência da pecuária e da agricultura. A região se destaca por ter culturas de lavoura permanente, como banana, manga, goiaba e coco, entre outras 30. Essas culturas são de grande relevância epidemiológica para a manutenção da fauna flebotomínica e ecossistema favorável aos reservatórios, por meio do predomínio de pastagens e matas ou florestas em vasta extensão territorial na bacia do Rio São Francisco.

Algumas limitações e vieses devem ser considerados na análise deste trabalho. Em primeiro lugar, os casos de LTA são provenientes de banco de dados secundários, o que pode levar a uma subestimação devido à subnotificação dos casos. Trata-se também de um estudo ecológico em que a unidade de análise é a população dos municípios dos circuitos e as variáveis utilizadas foram medidas de forma agrupada. Uma das limitações dos estudos ecológicos consiste na incapacidade de associar a exposição e a doença no nível individual, portanto, representa níveis de exposição média da população exposta. Os dados são provenientes de diferentes fontes e isto pode significar qualidade variável da informação 31 .

Outro ponto importante foi o uso de indicadores mais atualizados, já que para a construção das análises foram utilizados dados do Censo Agropecuário de 2006, do Censo Demográfico de 2010, do IMRS e IFDM, cujo ano base é 2010. Dessa forma, assumimos uma interação constante do processo entre a ocorrência de casos de LTA e dos indicadores no decorrer do tempo, apesar de reconhecermos o dinamismo da transmissão no espaço e no tempo. Destaca-se ainda, a dificuldade do uso de indicadores em apresentar concomitantemente todo o conjunto de propriedades desejáveis no estudo.

Outra limitação foi a não utilização de alguns fatores importantes, tais como clima (umidade, temperatura e precipitação), relevo e população de flebotomíneos, que por sua vez guardam relações diretas com ocorrência de casos de LTA 7,21,22,25. Efeitos negativos na variabilidade climática sobre as culturas, acompanhados pelo aumento da exploração dos recursos, podem ter grande impacto sobre a transmissão, porém, não foi objeto deste trabalho avaliar esta relação.

A LTA apresenta um ciclo complexo, dinâmico e na avaliação espaço-temporal, em que o ambiente constituído por áreas com paisagem variada, as formas de uso e ocupação do solo e os fatores socioeconômicos implicam processos ecológicos e sociais que resultam em influências significativas sobre a sua ocorrência no estado, cujos conhecimentos disponíveis sobre o assunto ainda são insuficientes para a explicação total do fenômeno em sua magnitude.

Maior incentivo para a realização de estudos sobre a exploração do espaço que permitam uma melhor definição das características ambientais, da paisagem local e do uso da terra, de forma associada com indicadores socioeconômicos é de grande importância na previsão de risco e para o apontamento correto das medidas de controle, conforme preconiza o Programa de Vigilância da Leishmaniose Tegumentar Americana proposto pelo Ministério da Saúde. 


\section{Colaboradores}

A. O. D. Temponi e M. X. Silva contribuíram com a concepção do projeto, análise e interpretação dos dados, e redação da versão final para aprovação. T. N. Cunha contribuiu com a revisão do artigo. M. G. Brito, M. L. Ferraz e S. A. Diniz contribuíram com a análise e interpretação dos dados, revisão do conteúdo intelectual e aprovação da versão final para publicação.

\section{Referências}

1. Secretaria de Vigilância em Saúde, Ministério da Saúde. Manual de vigilância da leishmaniose tegumentar americana. Versão eletrônica. http://bvsms.saude.gov.br/bvs/publicacoes/ manual_vigilancia_leishmaniose_tegumentar. pdf (acessado em 09/Mai/2017).

2. Fundação Nacional de Saúde. Vigilância e monitoramento da leishmaniose tegumentar americana em unidades territoriais - Brasil, 19942001. Brasília: Ministério da Saúde; 2002.

3. Secretaria de Vigilância em Saúde, Ministério da Saúde. Saúde Brasil 2011: uma análise da situação de saúde e a vigilância da saúde da mulher. Brasília: Editora MS; 2012.

4. Temponi AOD. Leishmaniose tegumentar americana. In: Secretaria de Estado de Saúde de Minas Gerais, organizador. Análise de situação de saúde de Minas Gerais. Belo Horizonte: Autêntica; 2012. p. 153-7.

5. Kawa H, Sabroza PC, Oliveira RM, Barcellos C. A produção do lugar de transmissão da leishmaniose tegumentar: o caso da Localidade Pau da Fome na cidade do Rio de Janeiro, Brasil. Cad Saúde Pública 2010; 26:1495-507.

6. Campbell-Lendrum D, Dujardim J-P, Martinez E, Feliciangeli MD, Perez JE, Silans LNMP, et al. Domestic and peridomestic transmission of American cutaneous leishmaniasis: changing epidemiological patterns present new control opportunities. Mem Inst Oswaldo Cruz 2001; 96:159-62.

7. Chaves LF, Cohen JM, Pascual M, Wilson ML Social exclusion modifies climate and deforestation impacts on a vector-borne disease. PLoS Negl Trop Dis 2008; 2:e176.

8. Rodríguez EM, Díaz F, Pérez MV. Spatio-temporal clustering of American cutaneous leishmaniasis in a rural municipality of Venezuela. Epidemics 2013; 5:11-9.

9. Passos VMA, Barreto SM, Romanha AJ, Krettli AU, Volpini AC, Gontijo CMF, et al. Leishmaniose tegumentar na Região Metropolitana de Belo Horizonte: aspectos clínicos, laboratoriais, terapêuticos e evolutivos (1989-1995). Rev Soc Bras Med Trop 2001; 34:5-12.

10. Gil JF, Nasser JR, Cajal SP, Juarez M, Acosta $\mathrm{N}$, Cimino RO, et al. Urban transmission of American cutaneous leishmaniasis in Argentina: spatial analysis study. Am J Trop Med Hyg 2010; 82:433-40

11. Negrão GN, Ferreira MEMC. Circuitos espaciais da leishmaniose tegumentar americana no Estado do Paraná. Hygeia 2013; 9:74-94.

12. Machado-Coelho GLL, Assunção R, Mayrink W, Caiaffa WT. American cutaneous leishmaniasis in Southeast Brazil: space-time clustering. Int J Epidemiol 1999; 28:982-9.

13. Instituto Brasileiro de Geografia e Estatística. Censo demográfico 2010. Característica da população e dos municípios. Resultados do universo. Rio de Janeiro: Instituto Brasileiro de Geografia e Estatística; 2010. 
14. Silva CR, Quintas MCL, Centeno JAS. Estudo dos métodos de interpolação do inverso da distância a uma potência. In: II Simpósio Brasileiro de Geomática e V Colóquio Brasileiro de Ciências Geodésicas. v. 1. Presidente Prudente: Universidade Estadual Paulista; 2007. p. 57-62.

15. Instituto Brasileiro de Geografia e Estatística. Censo agropecuário 2006. Rio de Janeiro: Instituto Brasileiro de Geografia e Estatística; 2006.

16. Teixeira IA. Incidência da tuberculose, índice de desenvolvimento humano e indicadores de vulnerabilidade familiar. Região Metropolitana de Belo Horizonte: uma abordagem multivariada [Tese de Doutorado]. Belo Horizonte: Universidade Federal de Minas Gerais; 2008.

17. Asensio LJ. Tecnicas de análisis de datos multidimensionales. Madrid: Ministério da Agricultura, Pesca y Alimentación; 1989.

18. Mingoti SA. Análise de dados através de métodos de estatística multivariada. Belo Horizonte: Editora UFMG; 2013.

19. Brito MG. O uso da terra e a distribuição espacial de casos humanos da síndrome cardiopulmonar por hantavírus em Minas Gerais, 1996 a 2007 [Tese de Doutorado]. Belo Horizonte: Escola de Veterinária, Universidade Federal de Minas Gerais; 2012.

20. Ocampo CB, Ferro MC, Cadena H, Gongora $\mathrm{R}$, Pérez M, Valderrama-Ardila $\mathrm{CH}$, et al. Environmental factors associated with American cutaneous leishmaniasis in a new Andean focus in Colombia. Trop Med Int Health 2012; 17:1309-17.

21. Valderrama-Ardila C, Alexander N, Ferro C, Cadena H, Marín D, Holford TR, et al. Environmental risk factors for the incidence of American cutaneous leishmaniasis in a subAndean zone of Colombia (Chaparral, Tolima). Am J Trop Med Hyg 2010; 82:243-50.

22. Dias ES, França-Silva JC, Silva JC, Monteiro EM, Paula KM, Gonçalves CM, et al. Flebotomíneos (Diptera: Psychodidae) de um foco de leishmaniose tegumentar no Estado de Minas Gerais. Rev Soc Bras Med Trop 2007; 40:49-52.
23. Nunes AG, Paula EV, Teodoro R, Prata A, Silva-Vergara ML. Aspectos epidemiológicos da leishmaniose tegumentar americana em Varzelândia, Minas Gerais, Brasil. Cad Saúde Pública 2006; 22:1343-7.

24. Cerbino Neto J, Werneck GL, Costa CHN. Factors associated with the incidence of urban visceral leishmaniasis: an ecological study in Teresina, Piauí State, Brazil. Cad Saúde Pública 2009; 25:1543-51.

25. Karagiannis-Voules D-A, Scholte RGC, Guimarães LH, Utzinger J, Vounatsou P. Bayesian geostatistical modeling of leishmaniasis incidence in Brazil. PLoS Negl Trop Dis 2013; 7:e2213.

26. Almeida AS, Werneck GL. Prediction of highrisk areas for visceral leishmaniasis using socioeconomic indicators and remote sensing data. Int J Health Geogr 2014; 13:13.

27. Basano SDA, Camargo LMA. Leishmaniose tegumentar americana: histórico, epidemiologia e perspectivas de controle. Rev Bras Epidemiol 2004; 7:328-37.

28. Confalonieri UE. Saúde na Amazônia: um modelo conceitual para a análise de paisagens e doenças. Estud Av 2005; 19:221-36.

29. Kawa H, Sabroza PC. Espacialização da leishmaniose tegumentar americana na cidade do Rio de Janeiro. Cad Saúde Pública 2002; 18:853-65.

30. Bastos SQDA, Gomes JE. Dinâmica da agricultura no Estado de Minas Gerais: análise diferencial-estrutural para o período 1994 a 2008. In: Anais do XIV Seminário sobre a Economia Mineira. http://econpapers.repec.org/book chap/cdpdiam10/009.htm (acessado em 29/ Mar/2014).

31. Medronho RA, Bloch KV, Luiz RR, Werneck GL. Epidemiologia. 2a Ed. São Paulo: Editora Atheneu; 2009. 


\section{Abstract}

The aim of this study was to explain the association between social-environmental factors and major land uses and the occurrence of cases of American tegumentary leishmaniasis (ATL) in the spatial circuits of production in Minas Gerais State, Brazil. This was an analytical-type ecological study based on secondary data on ATL divided by three-year period from 2007 to 2011, in which the analytical units were municipalities belonging to the spatial circuits. Two distinct stages were performed. The first was the elaboration of thematic maps with identification of the circuits. In the second, a new indicator, ATL cases by population density, was associated with social-environmental indicators and major land uses, submitted to multivariate principal components analysis (PCA). During the periods studied, three circuits were identified, distributed in the major regions of Northern Minas Gerais, Rio Doce Valley, and Greater Metropolitan Belo Horizonte. There was a strong association between ATL by population density and temporary crops, natural pasture, natural forest, unusable lands, and rural population, and a weak association with planted pasture. The association of cases with the major land uses variable in different agricultural profiles shows the occupational nature of ATL, associated mainly with rural workers. The association of the disease with environmental variables and deficient basic sanitation also proved relevant in the transmission profile in spatial circuits of production in Minas Gerais.

Cutaneous Leishmaniasis; Environmental Indicators; Spatial Analysis

\section{Resumen}

El objetivo de este estudio fue explicar la asociación de los factores socioambientales y explotación a gran escala de la tierra, con la ocurrencia de casos de leishmaniasis tegumentaria americana (LTA) en los circuitos espaciales de producción, en el Estado de Minas Gerais, Brasil. Se trata de un estudio ecológico de tipo analítico, basado en datos secundarios de casos de LTA, dividido por trienios, en el período entre 2007 a 2011, cuyas unidades de análisis fueron los municipios pertenecientes a los circuitos espaciales. Se realizaron dos etapas distintas, consistiendo la primera en la elaboración de mapas temáticos con la identificación de los circuitos, y la segunda etapa en un nuevo indicador de casos de LTA por densidad demográfica, que se asoció con indicadores socioambientales $y$ a dos explotaciones a gran escala de la tierra, sometidos a un análisis multivariado de componentes principales (ACP). Para el período evaluado, se identificaron tres circuitos distribuidos en las mesorregiones del norte de Minas Gerais, Vale do Rio Doce y región metropolitana de Belo Horizonte. Hubo una fuerte asociación de los casos de LTA por densidad demográfica con el trabajo temporal, pasto natural, bosque natural, tierras inaprovechables y población rural, y una escasa asociación con pasto plantado. La asociación de casos con variables de las explotaciones a gran escala de la tierra, con diferentes perfiles agropecuarios, demuestra el carácter ocupacional de la LTA, asociado principalmente a trabajadores de la zona rural. La asociación de la enfermedad con variables ambientales $y$ deficiencia en las condiciones de saneamiento básico, también demuestran relevancia en el perfil de transmisión en los circuitos espaciales de producción en Minas Gerais.

Leishmaniasis Cutánea; Indicadores Ambientales; Análisis Espacial
Recebido em 26/Set/2016

Versão final reapresentada em 05/Jun/2017 Aprovado em 27/Jul/2017 